\title{
MODULE OF A GEODESIC FOLIATION ON THE FLAT TORUS
}

\author{
Anna Kaźmierczak \\ Faculty of Mathematics and Computer Science \\ University of Lodz, Poland \\ akaz@math.uni.lodz.pl
}

\begin{abstract}
We study properties of geodesic foliations on the flat, n-dimensional torus. Using the isomorphism of the Hodge star, we obtain some facts concerning compact totally geodesic surfaces (which are the leaves of geodesic foliations). We compute the p-module of a geodesic foliation. On the basis of these results, we derive a kind of reciprocity formula for the product of modules of two orthogonal foliations. We relate this product with the number of intersections of their leaves. We also obtain a formula for a product of modules of a finite number of geodesic foliations.
\end{abstract}

Keywords: flat torus, module of a family of submanifolds, foliation, geodesics

\section{Introduction}

We study properties of geodesics of the flat, n-dimensional torus, which helps us to investigate relations between geodesic foliations on this manifold. The leaves of such a foliation have identical measures, since they are projections of the parallel $\mathrm{k}$-dimensional planes of $\mathbb{R}^{n}$. We introduce the notion of a closing k-plane of $\mathbb{R}^{n}$ (which can be treated as an element of the Grassmann algebra), that corresponds to a compact totally geodesic (ctg) surface, being a leaf of a geodesic foliation. An important concept that appears in the first section of the paper is a fundamental domain, which is a parallelepiped in a k-plane that has the same measure as the leaves of the corresponding geodesic foliation. It is convenient to study ctg surfaces with the aid of algebraic tools. A simple k-node (that is a specific element of the space $\Lambda_{k}\left(\mathbb{R}^{n}\right)$ ) can be identified with a closing k-plane. Since the Hodge star acting on a multi vector produces an orthogonal multi vector, we use this concept to obtain interesting facts concerning ctg surfaces. The main results are: Theorem 17, which actually claims that a totally geodesic surface orthogonal to a ctg surface (of complementary dimen- 
sion) is a ctg surface as well (it is also compact), and Theorem 29, saying that such surfaces have identical measures.

The second section concentrates on the p-module of a geodesic foliation. In the case of the flat torus, there is a simple formula for that quantity (Theorem 34). One can notice that for the unit torus, it is independent of the exponent $\mathrm{p}$. On the basis of the results of the previous section, we obtain an interesting relation between the modules of a pair of orthogonal geodesic foliations (Theorem 38). Their product depends directly on the number of intersections of the leaves of these foliations. This result can be understood as an extension of the reciprocity formula for a pair of conjugate foliations (see [1]). We also obtain a formula for a product of modules (with conjugate exponents) of a number of geodesic (not necessarily orthogonal) foliations (Corollary 37). It depends exclusively on the measures of the leaves of these foliations.

\section{Properties of closing k-planes}

First we introduce the necessary terminology.

Definition 1. A n-dimensional (unit) flat torus is the quotient manifold $M=\mathbb{R}^{n} / \mathbb{Z}^{n}$.

We take on $M$ the strongest topology in which the canonical projection $\pi: \mathbb{R}^{n} \rightarrow M$ is continuous. Then $\mathbb{R}^{n}$ is a covering of $M$. Therefore it is locally homeomorphic to an open set in $\mathbb{R}^{n}$ and the differential structure on $M$ can be defined by these homeomorphisms. The canonical projection also carries to $M$ the Riemannian structure from $\mathbb{R}^{n}$. We will use the same symbol $\mu_{n}$ for the Lebesgue measure defined on $\mathbb{R}^{n}$ and on $M$.

Definition 2. A node is a vector $v=\left(v_{1}, . ., v_{n}\right) \in \mathbb{Z}^{n}$. An irreducible node $v=\left(v_{1}, . ., v_{n}\right)$ is such a node that $G C D\left(v_{1}, . ., v_{n}\right)=1$ (we use the abbreviation $G C D$ for the greatest common divisor).

Let $v_{1}, \ldots, v_{k} \in \mathbb{R}^{n}$ be linearly independent. By a k-dimensional parallelepiped $R\left(v_{1}, \ldots, v_{k}\right)$ we call the set

$$
R\left(v_{1}, \ldots, v_{k}\right)=\left\{\alpha_{1} v_{1}+\ldots+\alpha_{k} v_{k}, \alpha_{1}, \ldots, \alpha_{k} \in[0,1)\right\} .
$$

We say that it is spanned by vectors $v_{1}, \ldots, v_{k}$ (if it is clear what vectors define a parallelepiped, it will be denoted simply by $R$ ).

Definition 3. We say that $R\left(v_{1}, \ldots, v_{k}\right)$ is a fundamental domain if $v_{1}, \ldots, v_{k}$ are linearly independent nodes and $R\left(v_{1}, \ldots, v_{k}\right)$ has the minimal $k$-dimensional Lebesgue measure from all $k$-dimensional parallelepipeds that are spanned by nodes and lie in $\operatorname{lin}\left(v_{1}, \ldots, v_{k}\right)$. 
Definition 4. A $\boldsymbol{k}$-plane is a $k$-dimensional plane that contains the origin. If a $k$-plane contains a set of $k$ linearly independent nodes it will be called a closing $\boldsymbol{k}$-plane.

By a direct computation we get

Lemma 5. Let $v_{1}, \ldots, v_{k} \in \mathbb{Z}^{n}$. Then $\left.\pi\right|_{R\left(v_{1}, \ldots, v_{k}\right)}: R\left(v_{1}, \ldots, v_{k}\right) \rightarrow \pi\left(\operatorname{lin}\left(v_{1}, \ldots, v_{k}\right)\right)$ is a surjection.

Lemma 6. Let $v_{1}, \ldots, v_{k} \in \mathbb{R}^{n}$ be linearly independent. If for certain different points $x_{1}, x_{2} \in R\left(v_{1}, \ldots, v_{k}\right)$ we have: $x_{1} \equiv x_{2}$, then $R\left(v_{1}, \ldots, v_{k}\right)$ contains a (nonzero) node.

As a corollary we obtain:

Lemma 7. Take $v_{1}, \ldots, v_{k} \in \mathbb{R}^{n}$. The projection

$$
\left.\pi\right|_{R\left(v_{1}, \ldots, v_{k}\right)}: R\left(v_{1}, \ldots, v_{k}\right) \rightarrow \pi\left(\operatorname{lin}\left(v_{1}, \ldots, v_{k}\right)\right)
$$

is injective if and only if $R\left(v_{1}, \ldots, v_{k}\right)$ does not contain a nonzero node.

The following auxiliary facts are easy to show, so we omit the proofs.

Lemma 8. Assume that a set $U \subset \mathbb{R}^{n}$ is such that $\left.\pi\right|_{U}$ is injective. Then for every $c \in \mathbb{R}^{n}$ also $\left.\pi\right|_{(U+c)}$ is injective (here: $U+c=\{x+c, x \in U\}$ ).

Lemma 9. If $R\left(v_{1}, \ldots, v_{k}\right)$ is spanned by nodes, then for arbitrary $z \in \operatorname{lin}\left(v_{1}, \ldots, v_{k}\right)$, $\pi(R+z)=\pi(R)$.

Lemma 10. For arbitrary parallelepiped $R$ and for every node $z \in \mathbb{Z}^{n}$, $\pi(R)=\pi(R+z)$.

Definition 11. Set $k>0$. An element of the vector space $\Lambda_{k} \mathbb{R}^{n}$ will be called a $\boldsymbol{k}$-vector (see[2]). Every $k$-vector $\hat{w}$ can be expressed uniquely as

$$
\hat{w}=\sum_{1 \leq i_{1}<\ldots<i_{k} \leq n} a_{i_{1}, \ldots, i_{k}} e_{i_{1}} \wedge \ldots \wedge e_{i_{k}},
$$

where $e_{1}, \ldots, e_{n}$ stands for the canonical basis of $\mathbb{R}^{n}$ and $a_{i_{1}, \ldots, i_{k}} \in \mathbb{R}$. We name these numbers the coefficients of the $\boldsymbol{k}$-vector $\hat{w}$ (with respect to the canonical basis). Moreover, the quantity:

$$
|\hat{w}|=\sqrt{\sum_{1 \leq i_{1}<\ldots<i_{k} \leq n}\left(a_{i_{1}, \ldots, i_{k}}\right)^{2}} .
$$

is the norm of $\Lambda_{k} \mathbb{R}^{n}$ (it can be shown that it does not depend on the choice of an orthonormal basis).

We know (see, for example, [3]) that the k-measure of a parallelepiped can be expressed by the coefficients of the exterior product of vectors that span it. Specifically: 
Lemma 12. For an arbitrary $k$-dimensional parallelepiped $R\left(v_{1}, \ldots, v_{k}\right)$,

$$
\mu_{k}\left(R\left(v_{1}, \ldots, v_{k}\right)\right)=\left|v_{1} \wedge \ldots \wedge v_{k}\right| .
$$

It is easy to show that a fundamental domain has the following important property (that also could serve as its definition).

Lemma 13. Let $v_{1}, \ldots, v_{k} \in \mathbb{Z}^{n}$ be linearly independent. A parallelepiped $R\left(v_{1}, \ldots, v_{k}\right)$ is a fundamental domain if and only if it does not contain a nonzero node.

Proof. $(\Rightarrow)$ Suppose the contrary - that $R$ is a fundamental domain and contains a nonzero node. In other words: there exist $z \in \mathbb{Z}^{n}$ and $\alpha_{1}, . ., \alpha_{k} \in[0,1)$ such that at least one of them is different from 0 and $z=\alpha_{1} v_{1}+. .+\alpha_{k} v_{k}$. Then

$$
\mu_{k}\left(R_{1}\right)=\left|v_{1} \wedge \ldots \wedge\left(\alpha_{1} v_{1}+. .+\alpha_{k} v_{k}\right)\right|=\alpha_{k}\left|v_{1} \wedge \ldots \wedge v_{k}\right|<\left|v_{1} \wedge \ldots \wedge v_{k}\right|=\mu_{k}(R)
$$

which would mean that $R$ is not a fundamental domain. Contradiction.

$(\Leftarrow)$ Assume that $R\left(v_{1}, \ldots, v_{k}\right)$ is spanned by nodes and does not contain a nonzero node. According to Lemma 7, $\varphi=\left.\pi\right|_{R}: R \rightarrow \operatorname{lin}\left(v_{1}, \ldots, v_{k}\right)_{\equiv}$ is injective. Moreover, it is a local isometry. Suppose that $R$ is not a fundamental domain and denote the fundamental domain of $\operatorname{lin}\left(v_{1}, \ldots, v_{k}\right)$ by $F$. Then $\mu_{k}(F)<\mu_{k}(R)$. From the already proved implication (" $\Rightarrow$ ") we know that a domain that contains a nonzero node is not fundamental. Therefore $F$ does not contain a (nonzero) node. So the mapping $\varphi_{1}=\left.\pi\right|_{F}: F \rightarrow \operatorname{lin}\left(v_{1}, \ldots, v_{k}\right)_{\equiv}$ has identical properties as $\varphi$. In consequence, the composition $\varphi^{-1} \circ \varphi_{1}: F \rightarrow R$ is a bijective local isometry. It is not true, however, since $\mu_{k}(F)<\mu_{k}(R)$. Contradiction.

The next lemma gives a useful characterization of a k-plane.

Lemma 14. For every $k$-plane $P \subset \mathbb{R}^{n}$ there exists such a permutation of the canonical coordinates from $\mathbb{R}^{n}$ and such an arrangement of numbers $\alpha_{i, j} \in \mathbb{R}$ (where $i=1, \ldots, n-k, j=1, \ldots, k)$, that every vector $v=\left(v^{1}, \ldots, v^{n}\right) \in P$ satisfies the following system of equations:

$$
\begin{array}{ccccc}
\alpha_{1,1} v^{1}+ & \ldots & +\alpha_{1, k} v^{k} & -v^{k+1} & =0 \\
\vdots & \vdots & \vdots & \vdots & \vdots \\
\alpha_{n-k, 1} v^{1}+ & \ldots & +\alpha_{n-k, k} v^{k} & -v^{n} & =0
\end{array}
$$

Definition 15. The numbers $\alpha_{i, j}$ will be called the slope coefficients of the $k$-plane $P$ (with respect to the mentioned permutation of coordinates).

The following result formulates a necessary and sufficient condition for the closeness of a k-plane. 
Lemma 16. A k-plane $P \subset \mathbb{R}^{n}$ is closing if and only if its slope coefficients are rational (for a certain permutation of coordinates and, in consequence, for every permutation for which these coefficients are finite).

Proof. $(\Rightarrow)$ The slope coefficients of $P$ can be computed from the system (2). For $i=1, \ldots, n-k$ we choose $\mathrm{k}$ linearly independent nodes $x_{1}, \ldots, x_{k}$. Substituting them to the i-th equation of (2), we get the system of linear equations with the unknowns $\alpha_{i, 1}, \ldots, \alpha_{i, k}$. Applying the Cramer formulas, it is easy to see that the solutions of this system are rational.

$(\Leftarrow)$ Assume that all the coefficients of $(2)$ are rational. Treating $\mathrm{k}$ first unknowns $x_{1}, \ldots, x_{k}$ as the parameters, we get the system of independent equations. Substituting integer numbers to these parameters, we obtain a rational solution $\left(x_{k+1}, \ldots, x_{n}\right)$ (using the Cramer formulas once more). Multiplying the vector $\left(x_{1}, \ldots, x_{n}\right)$ by the proper number, we get a node from $P$. Repeating this procedure for $k$ linearly independent sequences of parameters gives us the thesis.

Subsequent result will be very useful.

Theorem 17. For an arbitrary closing k-plane $P \subset \mathbb{R}^{n}, P^{\perp}$ is also closing.

Proof. According to (2), we can write that $P$ consists of the vectors of the form:

$$
\left(x^{1}, \ldots, x^{k}, \alpha_{1,1} x^{1}+\ldots+\alpha_{1, k} x^{k}, \ldots, \alpha_{n-k, 1} x^{1}+\ldots+\alpha_{n-k, k} x^{k}\right) .
$$

It is easy to see, that every vector from the following arrangement of $n-k$ linearly independent vectors:

$$
\begin{gathered}
\left(-\alpha_{1,1}, \ldots,-\alpha_{1, k}, 1,0, \ldots, 0\right), \\
\vdots \\
\left(-\alpha_{n-k, 1}, \ldots,-\alpha_{n-k, k}, 0, \ldots, 0,1\right),
\end{gathered}
$$

is orthogonal to $P$ - so these vectors span $P^{\perp}$. Since the slope coefficients $\alpha_{i, j}$ are rational, then multiplying every vector from the arrangement (3) by an adequately chosen integer number, we obtain $n-k$ linearly independent nodes from $P^{\perp}$.

An obvious consequence of the latter fact is

Corollary 18. If a $k$-plane $P$ has rational slope coefficients, then also (n-k)-surface $P^{\perp}$ has this property.

Definition 19. A $\boldsymbol{k}$-node is a $k$-vector that has integer coefficients. If the GCD of these coefficients equals one, we name it an irreducible $\boldsymbol{k}$-node. 
Definition 20. A $k$-vector that is the exterior product of $k$ certain vectors of $\mathbb{R}^{n}$ is called simple. Every such a $k$-vector determines the k-plane

$$
P=\left\{v \in \mathbb{R}^{n}: v \wedge \hat{w}=0\right\},
$$

that will be named the $\boldsymbol{k}$-plane generated by that $\boldsymbol{k}$-vector.

It is easy to see that (simple) k-vectors that generate the same k-plane must be linearly dependent.

The following implication holds

Theorem 21. Let $v_{1}, . ., v_{k} \in \mathbb{R}^{n}$ be linearly independent nodes whose exterior product $v_{1} \wedge \ldots \wedge v_{k}$ is an irreducible $k$-node. Then $R\left(v_{1}, \ldots, v_{k}\right)$ is a fundamental domain.

Proof. Suppose that $R\left(v_{1}, \ldots, v_{k}\right)$ is not a fundamental domain. Thus, there exist nodes $w_{1}, \ldots, w_{k}$ and a parallelepiped $R_{1}\left(w_{1}, \ldots, w_{k}\right)$ such that $\mu_{k}\left(R_{1}\right)<\mu_{k}(R)$. Obviously, $w_{1} \wedge \ldots \wedge w_{k}$ generates the same k-plane as $v_{1} \wedge \ldots \wedge v_{k}$, so it has the form $c v_{1} \wedge \ldots \wedge v_{k}$, where $c \in(0,1)$. Contradiction.

Recall the well-known formula for the coefficients of a simple k-vector:

Lemma 22. Assume that $b_{1}, \ldots, b_{n}$ is an orthonormal basis of $\mathbb{R}^{n}$ and let $v_{1}, \ldots, v_{k} \in R^{n}$ be an arrangement of arbitrary $k$-vectors (whose coefficients are written in that basis). Denote by $a_{i_{1}, \ldots, i_{k}}\left(1 \leq i_{1}<\ldots<i_{k} \leq n\right)$ the coordinates of the $k$-vector $v_{1} \wedge \ldots, \wedge v_{k}$ in that basis, and by $M_{i_{1}, \ldots, i_{k}}$-determinants of the matrix that consists of columns $i_{1}, \ldots, i_{k}$ of the matrix $\left(\begin{array}{c}v_{1} \\ \vdots \\ v_{k}\end{array}\right)$. Then

$$
M_{i_{1}, \ldots, i_{k}}=a_{i_{1}, \ldots, i_{k}} \text {. }
$$

Applying the latter result, we get:

Lemma 23. The k-plane generated by a simple k-node is closing.

Proof. We will show that the slope coefficients $\alpha_{j i}(i=1, \ldots, k, j=1, \ldots, n-k)$ of this plane are rational. Since $\hat{w}$ is simple, so there exist vectors $v_{1}, \ldots, v_{k}$, such that $v_{1} \wedge \ldots \wedge v_{k}=\hat{w}$. Define the matrix $M=\left(\begin{array}{c}v_{1} \\ \vdots \\ v_{k}\end{array}\right)$ and let $M_{i_{1}, \ldots, i_{k}}, 1 \leq i_{1}<\ldots<i_{k} \leq n$ be the determinant of the matrix built from these columns of $M$, that have numbers $i_{1}, \ldots, i_{k}$. Without loss of generality, we can assume that $M_{1, \ldots, k} \neq 0$. For arbitrary $i \in\{1, . ., k\}, j \in\{1, \ldots, n-k\}$,

$$
M_{1, \ldots, i-1, i+1, \ldots, k, k+j}=(-1)^{k-i} \alpha_{j i} M_{1, \ldots, k} .
$$


According to the previous lemma, the coefficients of the k-vector $\hat{w}$ are the minors of the maximal degree of $M$. From the assumption that $\hat{w}$ is a k-node, $M_{1, \ldots, k}=a_{1, \ldots, k} \in \mathbb{Z}$ and $\pm \alpha_{j i} M_{1, \ldots, k}=M_{1, \ldots, i-1, i+1, \ldots, k, k+j}=a_{1, \ldots, i-1, i+1, \ldots, k, k+j} \in \mathbb{Z}$. Therefore $\alpha_{j i} \in \mathbb{Q}$. From Lemma 16 we get the thesis.

On the basis of that fact, we obtain

Theorem 24. The following conditions are equivalent:

1. The exterior product of $k$ nodes that span a fundamental domain is an irreducible $k$-node.

2. For an arbitrary simple $k$-node $\hat{w}$ there exist such nodes $v_{1}, \ldots, v_{k}$, that $v_{1} \wedge \ldots \wedge v_{k}=\hat{w}$.

Proof. $1 . \Rightarrow 2$. Take any simple k-node $\hat{w}$. According to Lemma 23, it spans a closing k-plane $P$. Choose the nodes $z_{1}, \ldots, z_{k}$ in $P$ such that $R\left(z_{1}, \ldots, z_{k}\right)$ is a fundamental domain. Obviously, $c z_{1} \wedge \ldots \wedge z_{k}=\hat{w}$ for a certain $c \in \mathbb{R} \backslash\{0\}$. From the assumption, $c \in \mathbb{Z}$. Substituting $v_{1}=c z_{1}, v_{2}=z_{2}, \ldots, v_{k}=z_{k}$ we get the thesis.

$2 . \Rightarrow 1$. Suppose that $\neg 1$. Thus, there exists a fundamental domain $R\left(v_{1}, \ldots, v_{k}\right)$, such that $v_{1} \wedge \ldots \wedge v_{k}=\hat{w}$ is an reducible k-node. Take such a $p \in \mathbb{N}$, that $p \mid \hat{w}$. Then the $\mathrm{k}$-node $\frac{1}{p} \hat{w}$ cannot be the exterior product of any k-nodes, since it would contradict the fact that $R$ is a fundamental domain. Therefore $\neg 2$.

Now we quote an important theorem ([3]) that will give us several conclusions:

Theorem 25. A system of linear equations (with integer coefficients)

$$
a_{r 1} x_{1}+\ldots+a_{r n} x_{n}=0, \quad(r=1, \ldots, m),
$$

where $m<n$, has $n-m$ solutions $x_{s}=\left(x_{s}^{1}, \ldots, x_{s}^{n}\right) \in \mathbb{Z}^{n}(s=1, \ldots, n-m)$, such that the matrix whose rows are the vectors of these solutions has a property that the GCD of its minors of maximal rank equals 1.

A consequence of the above result is the implication converse to that of Theorem 21:

Corollary 26. The exterior product of the vectors that span the fundamental domain (of a closing k-plane P) is an irreducible k-node.

Proof. Since $P$ is closing, then, from Lemma 16, all its slope coefficients are rational. Thus, the system of equations determining $P$ can be transformed to (5), for $m=n-k$. From the previous theorem, we know that it has $k$ solutions $w_{1}, \ldots, w_{k}$, such that minors of the rank $k$ of the matrix composed from these solutions constitute a set of relatively prime numbers. Therefore, from Lemma 22 , the k-node $w_{1} \wedge \ldots \wedge w_{k}$ is irreducible. According to Theorem $21, R\left(w_{1}, \ldots, w_{k}\right)$ is a fundamental domain. At last, from the definition of the latter and Lemma 12 results that every fundamental domain of $P$ has to be irreducible. 
On the account of Theorem 24, the above conclusion implies the following

Corollary 27. For an arbitrary simple $k$-node $\hat{w}$, there exist nodes $v_{1}, \ldots, v_{k} \in \mathbb{R}^{n}$ such that $v_{1} \wedge \ldots \wedge v_{k}=\hat{w}$.

Definition 28. Let $a_{i_{1}, \ldots, i_{k}} \in \mathbb{R}\left(1 \leq i_{1}<\ldots<i_{k} \leq n\right)$ be the coefficients of a $k$-vector $\hat{w}$ (with respect to the canonical basis). The Hodge star of $\hat{w}$ is a $(n-k)$-vector $* \hat{w}$ of the form (see [4]):

$$
* \hat{w}=\sum_{1 \leq i_{1}<\ldots<i_{k} \leq n} a_{i_{1}, \ldots, i_{k}} \operatorname{sgn}\left(i_{1}, \ldots, i_{k}, j_{1}, \ldots, j_{n-k}\right) e_{j_{1}} \wedge \ldots \wedge e_{j_{n-k}},
$$

where $j_{1}, \ldots, j_{n-k} \in\{1, \ldots, n\} \backslash\left\{i_{1}, \ldots, i_{k}\right\}, 1 \leq j_{1}<\ldots<j_{n-k} \leq n$.

Finally, as a consequence of the preceding facts, we obtain

Theorem 29. Assume that $P \subset \mathbb{R}^{n}$ is a closing $k$-plane and the nodes $v_{1}, \ldots, v_{k}$ span a fundamental domain in $P$ and that $v_{1}^{\prime}, \ldots, v_{n-k}^{\prime}$ are the nodes that span such a domain in $P^{\perp}$. Then $*\left(v_{1} \wedge \ldots \wedge v_{k}\right)= \pm v_{1}^{\prime} \wedge \ldots \wedge v_{n-k}^{\prime}$ and

$$
\mu_{k}\left(R\left(v_{1}, \ldots, v_{k}\right)\right)=\mu_{n-k}\left(R\left(v_{1}^{\prime}, \ldots, v_{n-k}^{\prime}\right)\right) .
$$

Proof. Let $\hat{w}=v_{1} \wedge \ldots \wedge v_{k}$. From the definition, $* \hat{w}$ is a (n-k)-node whose coefficients are - in the absolute value - equal to the corresponding coefficients of $\hat{w}$. Thus, $* \hat{w}$ is irreducible. Moreover, $* \hat{w}=c v_{1}^{\prime} \wedge \ldots \wedge v_{n-k}^{\prime}$ for a certain $c \in \mathbb{R}$. However, from the Conclusion 26, $v_{1}^{\prime} \wedge \ldots \wedge v_{n-k}^{\prime}$ is irreducible as well. So $|c|=1$ and we get the first part of the thesis. Its second part follows from Lemma 12.

The reasoning presented above can be repeated also for a more general flat torus, defined by replacing in Definition 1 the module $\mathbb{Z}^{n}$ by the module generated by a given basis of $\mathbb{R}^{n}$ (that can be named the basis of torus). In this case a node (Def. 2) should be defined as a vector that has integer coefficients with respect to the basis of a torus. Analogically, in the definition of a k-node (Def. 27) the canonical basis should be replaced by the basis of torus. The same modification would be applied in the definition of the slope coefficients of a k-plane. Taking those changes into consideration, all the results that precede Theorem 17 hold (assuming that $v \in \mathbb{Z}^{n}$ denotes a vector having integer coordinates in the basis of torus), whereas that theorem itself is true if only the vectors that constitute the basis of torus have rational coordinates (in the canonical basis). Other results, excluding Lemma 12 and Theorem 29, transform automatically to another flat torus. Lemma 12 is true in its original form - the coefficients of a k-node related with the fundamental domain should be written in the canonical basis. In turn, the Theorem 29 holds only for the unit (flat) torus, since the coefficients in the formula (6) for the Hodge star are taken with respect to the canonical basis. 


\section{Module on the flat torus}

Set $0<k<n$. Recall that

Definition 30. Denote by $\mathscr{M}$ a family of smooth, $k$-dimensional submanifolds of $M$. We call the function $f$-admissible $(p \geq 1)$ for $\mathscr{M}$ with respect to $M$ (writing: $\left.f \in \operatorname{adm}_{p}(\mathscr{M}, M)\right)$ if

1. $f \in L^{p}(M)$

2. $f \geq 0$ almost everywhere on $M$

3. $\int_{L} f d \mu_{k} \geq 1$ for almost every element $L \in \mathscr{M}$.

The p-module (or the module with exponent $p$ ) of a family $\mathscr{M}$ of smooth, $k$-dimensional submanifolds of $M$ is a number:

$$
\bmod _{p}(\mathscr{M}, M)=\inf _{f \in \operatorname{adm}_{p}(\mathscr{M}, M)}\|f\|_{L^{p}(M)},
$$

(setting: $\bmod _{p}(\mathscr{M}, M)=\infty$ if adm $\left.(\mathscr{M}, M)=\emptyset\right)$.

A p-admissible function $f_{0}$ is called p-extremal if

$$
\left\|f_{0}\right\|_{L^{p}(M)}=\bmod _{p}(\mathscr{M}, M) .
$$

Definition 31. ([5]) A k-dimensional foliation is a decomposition of $M$ into a family $\mathscr{F}$ of disjoint, connected submanifolds of dimension $k$ with the property that for every point $x \in M$ there exists a neighborhood $D$ of $x$ and a chart $\varphi=\left(\varphi^{1}, \varphi^{2}, \ldots, \varphi^{n}\right): D \rightarrow \mathbb{R}^{n}$, such that $\varphi(D)$ is an open cube and for every $L \in \mathscr{F}$, satisfying: $L \cap D \neq 0$,

$$
\begin{aligned}
& \varphi_{\mid L}^{1}=\text { const }, \quad \varphi_{\mid L}^{j+k+1}=\text { const }, \\
& \begin{array}{ll}
\vdots & \text { and } \quad \vdots \\
\varphi_{\mid L}^{j}=\text { const }, & \\
\varphi_{\mid L}^{n}=\text { const },
\end{array}
\end{aligned}
$$

for a given $j \in\{0, \ldots, n-k\}$. The elements of $\mathscr{F}$ are called leaves.

The next theorem ([6]) presents a useful formula for a module of a foliation defined by a submersion (i.e. a foliation whose leaves are the level sets of that submersion).

Theorem 32. If a foliation $\mathscr{F}$ of $M$ defined by a submersion $\phi$ and for a.e. $L$, $\int_{L} J_{\phi}^{\frac{1}{p-1}} d \mu_{k}<\infty$, then for $p>1$

$$
\bmod _{p}^{p}(\mathscr{F})=\int_{\phi(M)}\left(\int_{L_{x}} J_{\phi}^{\frac{1}{p-1}} d \mu_{L_{x}}\right)^{1-p} d \mu_{\phi(M)},
$$


and if $\bmod _{p}^{p}(\mathscr{F})<\infty$, there exists exactly one extremal function $v: M \rightarrow \mathbb{R}$,

$$
v(x)=\frac{J_{\phi}(x)^{\frac{1}{p-1}}}{\int_{L_{x}} J_{\phi}^{\frac{1}{p-1}} d \mu_{L_{x}}},
$$

where $J_{\phi}$ is a function that assigns to every $x \in M$ an absolute value of the determinant of the matrix of the isomorfism: $\left.\phi_{*}(x)\right|_{k e r\left(\phi_{*}(x)\right)^{\perp}}$ (in orthonormal bases).

Definition 33. A totally geodesic ( $k$-dimensional) surface is such a $k$-dimensional surface $G \subset M$, that every geodesic curve tangent to $G$ in one point, lies in $G$. A compact totally geodesic surface will be briefly named a ctg surface.

A compact geodesic ( $k$-dimensional) foliation on $M$ is a foliation whose leaves are $k$-dimensional ctg surfaces of $M$.

On the basis of Theorem 32, we compute the module of a geodesic foliation:

Lemma 34. Let $\mathscr{F}$ be a compact geodesic foliation on $M$. Then

$$
\bmod _{p}(\mathscr{F})=\frac{\mu_{n}(M)^{\frac{1}{p}}}{v}
$$

for $p>1$, where $v$ denotes the measure of a leaf of $\mathscr{F}$

Proof. According to Theorem 32, $\mathscr{F}$ has the unique extremal function, that equals $\frac{1}{v}$. Thus

$$
\bmod _{p}(\mathscr{F})=\left(\int_{M}\left(\frac{1}{v}\right)^{p} d \mu_{n}\right)^{\frac{1}{p}}=\frac{\mu_{n}(M)^{\frac{1}{p}}}{v}
$$

In particular, it is easy to see that the module of such a foliation on a unit torus does not depend on the exponent $p$.

Moreover, we get the following formula for the product of their modules with conjugate exponents:

Theorem 35. Let $\mathscr{F}_{1}, . ., \mathscr{F}_{k}$ be compact geodesic foliations on $M$. For arbitrary conjugate exponents $p_{1}, \ldots, p_{k}$,

$$
\bmod _{p_{1}}\left(\mathscr{F}_{1}\right) \cdot \bmod _{p_{2}}\left(\mathscr{F}_{2}\right) \cdot \ldots \cdot \bmod _{p_{k}}\left(\mathscr{F}_{k}\right)=\frac{\mu_{n}(M)}{v_{1} \cdot \ldots \cdot v_{k}},
$$

where $v_{1}, \ldots, v_{k}$ denote the measures of the leaves of the corresponding foliations.

Proof. This is a straightforward consequence of Lemma 34.

Since now, we assume that $M$ is the unit flat torus. 
Lemma 36. Let $R$ be a $n$-dimensional parallelepiped spanned by the nodes $v_{1}, \ldots, v_{n}$. Assume that $R$ has an integer volume $v \in \mathbb{N}$. Then $R$ contains exactly $v$ representatives of every equivalence class of $M$.

Proof. Let $\bar{M}$ be the torus generated by the basis $v_{1}, \ldots, v_{k}$. Denote the equivalence relation defining $\bar{M}$ by $\equiv_{\bar{M}}$. Choose an arbitrary $x \in \mathbb{R}^{n}$ and denote by $x_{1}, \ldots, x_{m} \in R$ such points, that $x_{1}, \ldots, x_{m} \in[x]$. Now, take any $y \in \mathbb{R}^{n}$. Let $s$ be such a point, that $y=x_{1}+s$. Define $y_{i}=x_{i}+s$, for $i=1, \ldots, m$. Then for every $i, y_{i} \in[y]$ (since $y_{i}-y=$ $\left.x_{i}-x_{1} \in \mathbb{Z}^{n}\right)$. Simultaneously, $\left[y_{1}\right]_{\equiv_{\bar{M}}} \neq \ldots \neq\left[y_{m}\right]_{\equiv_{\bar{M}}}$ (since $\left[x_{1}\right]_{\equiv_{\bar{M}}} \neq \ldots \neq\left[x_{m}\right]_{\equiv_{\bar{M}}}$ ). Next, from the definition of $\bar{M}$ it results that for every $z \in \mathbb{R}^{n}$, there exists $\tilde{z} \in R$, such that $\tilde{z} \in[z]_{\equiv_{\bar{M}}}$. So, there exist points $\tilde{y}_{i} \in\left[y_{i}\right]_{\equiv_{\bar{M}}}$, satisfying: $\tilde{y}_{i} \in R$. From the arbitrariness of $x$ and $y$ we obtain that $R$ contains the same number of representatives of every equivalence class of $M$. From the fact that $J_{\pi}=1$ we get the thesis $(m=v)$.

An immediate consequence of this lemma is

Corollary 37. A parallelepiped of the measure $n$, that is spanned by nodes, contains exactly $n-1$ nonzero nodes.

We will show a relation between the product of modules of two orthogonal geodesic foliations with the number of intersections of their leaves.

Theorem 38. Fix $1 \leq k<n$. Assume that $\mathscr{G}_{1}$ is a $k$-dimensional compact geodesic foliation on $M$, whereas $\mathscr{G}_{2}$ is a $(n-k)$-dimensional geodesic foliation, whose leaves are orthogonal to the leaves of $\mathscr{G}_{1}$. Then, for $p>1$,

$$
\bmod _{p}\left(\mathscr{G}_{1}\right) \cdot \bmod _{p}\left(\mathscr{G}_{2}\right)=\frac{1}{I},
$$

where I denotes the number of intersections of an arbitrary leaf of $\mathscr{G}_{1}$ with an arbitrary leaf of $\mathscr{G}_{2}$.

Proof. Let $F_{1}, F_{2}$ be fundamental domains of the (k- and (n-k)-) planes covering the leaves of $G_{1} \in \mathscr{G}_{1}$ that go through [0]. Denote $v=\mu_{k}\left(F_{1}\right)=\mu_{(n-k)}\left(F_{2}\right)$ (this equality results from the Conclusion 29). Define $R=F_{1} \times F_{2}$. Then $\mu_{n}(R)=v^{2}$. From the Conclusion 37 we know that $R$ contains exactly $v^{2}$ nodes (including 0 ). Denote them by $w_{1}, \ldots, w_{v^{2}}$. Let $F_{1}^{i}=F_{1}+c_{i}$, for $i=1, \ldots, v^{2}$, where $c_{i} \in F_{2}$ is such a (k-dimensional) parallelepiped, that $w_{i} \in F_{1}^{i}$. From Lemmas 9 and 10 we have that for every $i$, the projection $\pi$ transforms $F_{1}^{i}$ bijectively on $G_{1}$. Now let $G_{2}$ be an arbitrary leaf of the foliation $\mathscr{G}_{2}$. From the definition of $F_{2}$ and the fact that $\pi(R)=M$, there exists a (n-k)-dimensional parallelepiped $F_{2}^{1}=F_{2}+c\left(c \in F_{1}\right)$, for which $\pi\left(F_{2}^{1}\right)=G_{2}$. The orthogonality of $F_{1}$ and $F_{2}$ ensures that $F_{2}^{1}$ intersects every $F_{1}^{i}$ exactly once. Denote the points of these intersections by $s_{i}, i=1, \ldots, v^{2}$. On the 
other hand, we know that $\left.\pi\right|_{F_{2}^{1}}$ is bijective (since $F_{2}^{1}$ is a translation of $F_{2}$ - see Lemma 8). Thus $\pi\left(s_{i}\right)$ are distinct points of $M$. In consequence, $G_{2}=\pi\left(F_{2}^{1}\right)$ intersects $G_{1}$ exactly $v^{2}$ times. The above argument, applied for $G_{1}$, can be repeated for an arbitrary leaf of $\mathscr{G}_{1}$. Application of formula (9) finishes the proof.

\section{Final remarks}

Theorem 38, that expresses the product of modules as a function of the number of intersections of the leaves of two orthogonal, geodesic foliations, is a consequence of the results present before: properties of compact totally geodesic surfaces on the flat torus and the formula for the module of a geodesic foliation. It is also interesting if any analogues of this relation can be found for other manifolds. It will be the subject of our further research.

\section{Acknowledgements}

The author would like to sincerely thank Andrzej Schinzel for his valuable remarks and indication of the literature. She also expresses her gratitude to Antoni Pierzchalski for suggesting the problem and discussions.

\section{References}

[1] Ciska M., Pierzchalski A., On the modulus of level sets of conjugate submersions, Differential Geometry and its Applications, to appear.

[2] Federer H., Geometric Measure Theory, Springer-Verlag, Berlin-Heidelberg -New York 1969.

[3] Skolem T.H, Diophantische Gleichungen, Verlag von Julius Springer, Berlin 1938.

[4] Warner F.W., Foundations of Differentiable Manifolds and Lie Groups, Springer-Verlag, Berlin 1983.

[5] Candel A., Conlon L., Foliations I. American Mathematical Society, Providence Rhode Island 2000.

[6] Pierzchalski A., The k-module of level sets of differential mappings, Scientific Communications of the Czechoslovakian-GDR-Polish School on Differential Geometry at Boszkowo (1978), Math. Inst. Polish Acad. Sci., Warsaw, 180-185 1979. 